\title{
Aspects of Confinement: a Brief Review
}

\author{
Eric S. Swanson \\ Department of Physics and Astronomy, University of Pittsburgh, Pittsburgh PA, 15260
}

\begin{abstract}
A brief and biased overview of the phenomenon of confinement in QCD is presented in three parts: (1) the definition of confinement, (2) properties of confinement, (3) ideas of confinement. The second part chiefly consists of a brief review of recent lattice computations related to confinement while the third summarizes some of the current analytical approaches to understanding confinement. These include the Dyson-Schwinger formalism in Landau gauge, Hamiltonian QCD in Coulomb gauge, and the vortex picture of confinement.
\end{abstract}

\section{DEFINITIONS OF CONFINEMENT}

Confinement is the poster boy of nonperturbative physics for good reason: it is associated with a linear potential with a string tension,

$$
\sigma \propto \Lambda^{2} \mathrm{e}^{-\int \frac{d g}{\beta(g)}}
$$

which is nonperturbative in the coupling.

Although this statement has intuitive appeal, one must be careful in defining confinement. For example, it is often loosely defined as the absence of free quarks in nature. But it is conceivable, and even possible, that there exists a coloured scalar particle which can form bound states with quarks. The resultant particles would then carry flavour and fractional electric charge[1], which is likely not the intent of the definition.

Similarly, requiring that all observable particles be colour singlets encompasses gauge theories in both the confinement and the Higgs phases. The latter also manifests colour singlet states because colour charge is completely screened.

One may hope to improve the situation by focussing on the expected physical properties of confinement. For example, the appearance of a long range linear potential between quarks is a reasonable requirement. Of course, the problem here is that string breaking will occur once the potential energy approaches the quark pair creation threshold. The conventional way out of this difficulty is to consider the work to separate two quarks as the quark masses approach infinity. Thus one is led to the strange position of defining quark confinement in a limit which removes quarks from the theory.

\section{Centre Vortices}

It is traditional to implement the last definition in lattice gauge theory with the aid of the Wilson loop: 


$$
\langle W L\rangle=\int D U_{\ell} \operatorname{tr}\left[\prod_{i \in C} U_{i}\right] \mathrm{e}^{-S_{Y M}}
$$

where $C$ is a large planar loop and the pure gauge action is given by

$$
S_{Y M}=\frac{6}{g^{2}} \sum_{P}\left(1-\frac{1}{N_{c}} \Re \operatorname{tr}\left[U_{P}\right]\right) .
$$

Here the gluonic degrees of freedom are represented by link variables $U_{\ell}=$ $\exp \left(i g T^{a} A_{\mu}^{a}(x)\right)$ where $\ell$ represents a link on a spacetime lattice which starts at the point $x$ and points in the $\hat{\mu}$ direction and the lattice spacing has been set to unity. The sum in the action is over plaquettes, $P$, which are the smallest closed loops permitted on the lattice. $U_{P}$ is a product of link variables around a plaquette and is thus a lattice implementation of the two-forms of gauge theories. It is measurements of the Wilson loop which have provided the most compelling demonstrations of the confinement phenomenon.
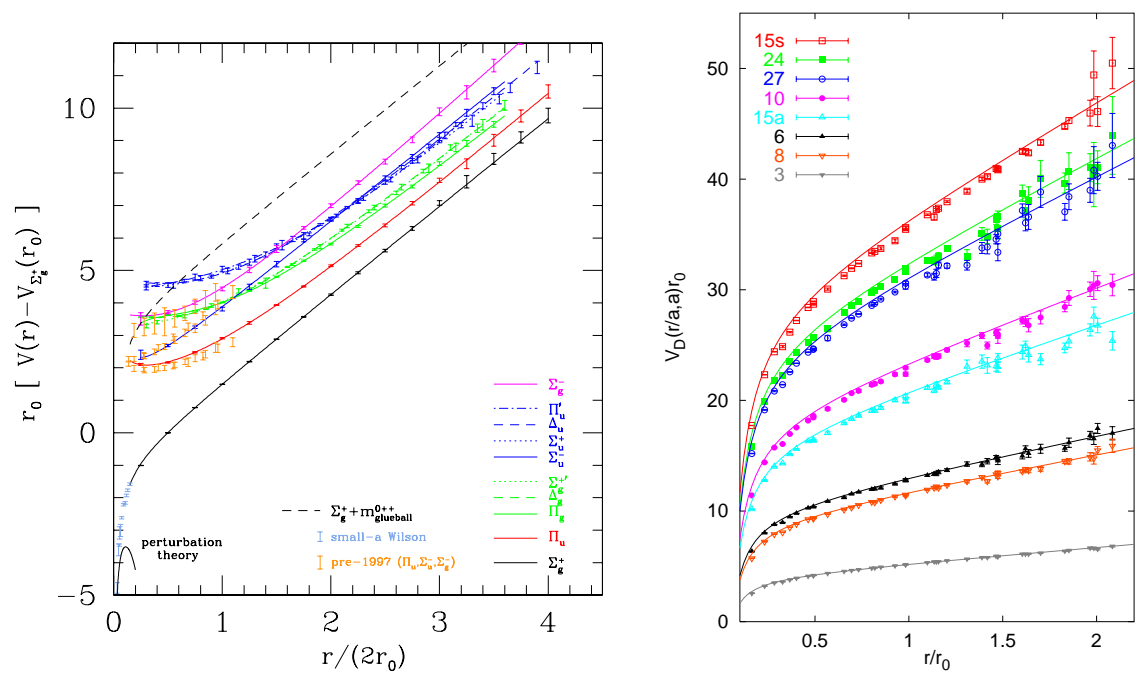

FIGURE 1. (left) Wilson Loop Measurements of Various Static Quark Potentials[2]. (right) Casimir Scaling of Confinement[9].

The lattice definition of QCD and confinement makes it especially easy to see the significance of the centre group of SU(3). The centre is defined as the set of all elements of the gauge group which commute with all other elements. Evidently these are given by the set $\left\{z=\exp \left(2 \pi n / N_{c}\right) \mathscr{I}\right\}$ where $n=0, \ldots, N_{c}-1$. This set forms the group $Z_{N_{c}}$. It is clear that multiplying all temporal link variables on a particular time slice by an element of the group, $U_{t}\left(t_{0}, \mathbf{x}\right) \rightarrow z U_{t}\left(t_{0}, \mathbf{x}\right)$ does not change the value of a plaquette, implying that QCD is invariant under global $Z_{N_{c}}$ transformations.

We now consider a Wilson loop which extends in the temporal direction for the entire length of the lattice (and, because of the imposition of boundary conditions, encircles the lattice), called a Polyakov line. If one makes the transformation discussed above it is clear that the Polyakov line picks up a phase: $P L(\mathbf{x}) \rightarrow z P L(\mathbf{x})$. Since the theory is invariant under such a transformation one must have either $\langle P L(\mathbf{x})\rangle=0$ if the vacuum 
of QCD shares the symmetry of the action or $\langle P L(\mathbf{x})\rangle \neq 0$ if the theory is in the $Z_{N_{c}}$ broken phase.

The connection with confinement arises once it is realized that the Polyakov line measures the free energy of a static quark at the position $\mathbf{x}:\langle P L(\mathbf{x})\rangle=\exp (-E T)$ where $T$ is the temporal extent of the lattice. But the free energy of an isolated confined quark is infinite, thus $Q C D$ must be in the unbroken $Z_{N_{c}}$ symmetry phase if it is confining.

It is useful to note that $Z_{N_{c}}$ transformations are an example of "singular gauge transformations" (which are not gauge transformations!), namely they may be generated by performing a gauge transformation which is periodic modulo a $Z_{N_{c}}$ phase factor:

$$
U_{t}(t, \mathbf{x}) \rightarrow g(t, \mathbf{x}) U_{t}(t, \mathbf{x}) g^{\dagger}(t+1, \mathbf{x})
$$

with $g(T+1, \mathbf{x})=z^{*} g(1, \mathbf{x})$. For example the symmetry transformation $U_{t}\left(t_{0}, \mathbf{x}\right) \rightarrow$ $z U_{t}\left(t_{0}, \mathbf{x}\right)$ may be achieved by setting $g(t, \mathbf{x})=1$ for $t \leq t_{0}$ and $g(t, \mathbf{x})=z^{*}$ for $t>$ $t_{0}$. Singular gauge transformations are of central interest to the study of confinement because the structures associated with them are often postulated to cause confinement. We shall return to this in section III below.

\section{Kugo-Ojima Criteria}

The final definition of confinement to be considered here was first investigated by Kugo and Ojima shortly after the invention of QCD[3]. Their starting assumption was that only BRST singlets may be allowed as physical states if confinement is to hold. A sufficient condition for this is that the ghost propagator in Landau gauge is enhanced in the infrared:

$$
D_{G}(k)=-\frac{1}{k^{2}} \frac{1}{1+u\left(k^{2}\right)} \equiv-\frac{G\left(k^{2}\right)}{k^{2}}
$$

with $u(0)=-1$. This may be related to the gluon propagator via Dyson-Schwinger equations and implies that there is an infrared suppression of the transverse gluon correlator:

$$
D_{\mu \nu}(k)=\frac{Z\left(k^{2}\right)}{k^{2}}\left(\delta_{\mu \nu}-\frac{k_{\mu} k_{v}}{k^{2}}\right)
$$

with $Z\left(k^{2}\right) / k^{2} \rightarrow 0$ as $k \rightarrow 0[4]$.

It is interesting to note that these criteria are consistent with results derived by Zwanziger in noncovariant gauges[6].

\section{PROPERTIES OF CONFINEMENT}

At present the only reliable method for determining properties of confinement is with lattice computations. Indeed, the detection of confinement (with the Wilson loop) was one of the first lattice computations and it continues as the standard bearer of lattice gauge theory. 
Recent investigations have revealed much beyond the static quark interaction. Perhaps the most famous examples are plots of action or field density which clearly show the formation of tubes of gluonic flux forming between the colour source and sink [7]. Of course, such tubes fit in very well with the naive notion of linear quark confinement. More interesting is recent investigations of the dynamics of flux tubes, as represented by the higher surfaces in Fig. 11left). These may be interpreted as adiabatic energy surfaces describing hybrid mesons.

Another type of flux tube investigation places small kinks in Wilson loops to study the spin-dependence of the long range confining force[8]. The results are consistent with the supposition that confinement is of a Lorentz scalar nature (it is important to note, however, that mapping the lattice results to this type of interaction is an effective, low energy approximation only).

\section{Casimir Scaling}

Figure 1(right) shows in a compelling way the property of Casimir scaling of confinement. The figure was obtained by measuring the Wilson loop for sources in various representations of SU(3). The interaction between colour triplets is the lowest surface in the figure and forms the template for the others. In the figure one sees higher surfaces with sources in the $8,6,15_{A}, 10,27,24$, and $15_{S}$ representations. The curves are obtained by multiplying a fit to the lowest (fundamental representation) surface by the quadratic Casimir, $\mathscr{C}_{R}^{2}=\left\langle R\left|T^{a} T^{a}\right| R\right\rangle$ divided by $\mathscr{C}_{F}^{2}$. The quadratic Casimir is given by $\left(p^{2}+q^{2}+p q\right) / 3+p+q$ where $(p, q)$ is the Dynkin index of the representation. The agreement is remarkable and is a strong indication that the colour structure of confinement may be written as

$$
\int \bar{\psi} T^{a} \psi \ldots \bar{\psi} T^{a} \psi
$$

where the ellipsis represents Lorentz and spatial dependence.

\section{String Behaviour}

In pre-QCD days strings were invoked as a fundamental theory of QCD. With the advent of QCD, strings have survived as an effective description of flux tubes. In particular one expects that

(i) transverse flux tube profiles should be logarithmically divergent with the tube length (due to string 'roughening');

(ii) the ground state potential should exhibit a universal 'Luescher term', $-\pi /(12 R)$;

(iii) the string excitation spectrum should be that of bosonic string modes, $\pi / R$.

Juge, Kuti, and Morningstar have carried out a detailed analysis of the relationship of the hybrid surfaces of Fig. 11left) to string excitations[10]. They have found that surface excitations only have $\pi / R$ splittings for very large source separation (roughly 4 fermi or greater); see Figure 2 (left). Furthermore, there is a cross over region at about 1 fermi where the surfaces move from a perturbative behaviour (characterized by the 'gluelump' spectrum) to a string-like behaviour.

Point (ii) has been examined with a new algorithm by Luescher and Weisz[11]; their main results are shown in Figure 2(right). One sees that the expected behaviour of the confinement potential is achieved at a source separation of roughly $1 / 2 \mathrm{fm}$ or less. String- 

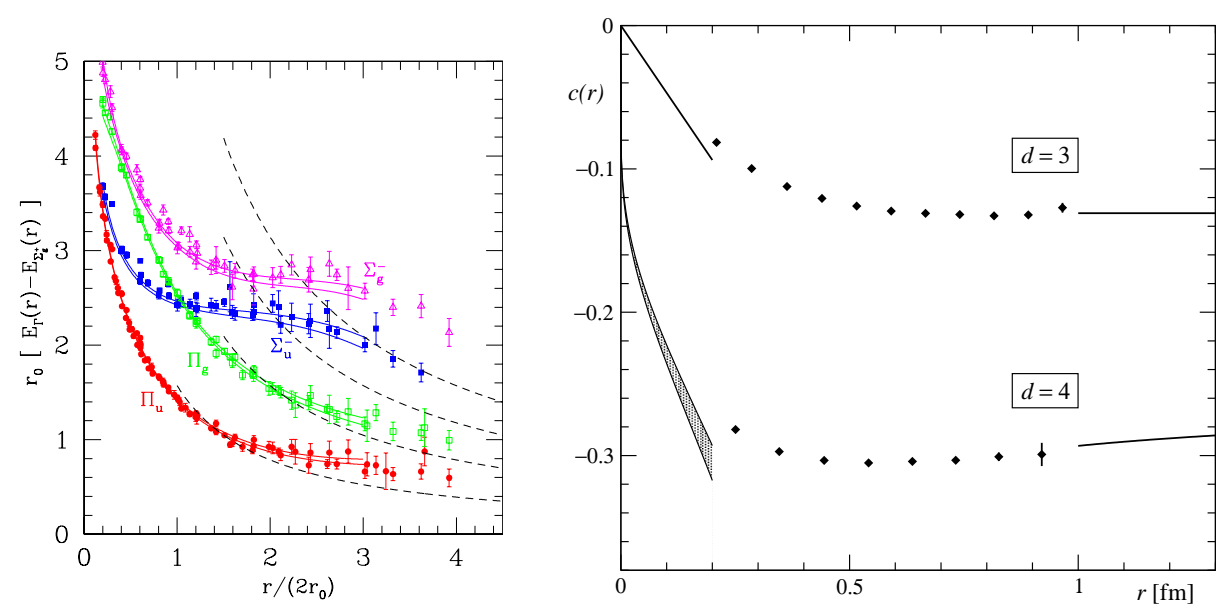

FIGURE 2. (left) Hybrid Surface Energy Differences. $2 r_{0}$ is roughly 1 fermi[10]. (right) The Luescher Term in $4-\mathrm{d}(-\pi / 12)$ and $3-\mathrm{d}(-\pi / 24)[11]$.

like behaviour at such short distances may be called precocious and is very difficult to understand in light of the conclusion of Juge, Kuti, and Morningstar. It appears that the existence of a $1 / r$ interaction with the expected Luescher coefficient is not necessarily indicative of string dynamics.

\section{Baryonic Flux Tubes}

Finally, investigations of the static baryon interaction have begun. The chief point of interest is whether the expected flux tubes form into a ' $Y$ ' shape or a ' $\Delta$ ' shape (ie., is the effective baryonic quark interaction two-body or three-body?). This may be addressed by carefully examining the baryonic energy under a variety of quark configurations. Figure 3 shows the flux tube which arises in one such configuration (which seems to be an interpolation of Y and $\Delta$ ). Current results are mixed, with some groups claiming support for the two-body hypothesis [13] and some for the three-body hypothesis[14]. Finally, a strong operator dependence in the flux tube profiles has been observed[15], which clearly needs to be settled before definitive conclusions can be reached.

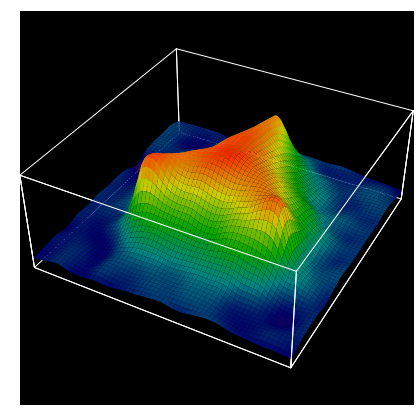

FIGURE 3. The Baryonic Flux Tube. The distance between the tube junction and a quark is approximately $1 / 2 \mathrm{fm}[12]$. 


\section{IDEAS OF CONFINEMENT}

The final portion of this report deals with analytic attempts to describe confinement. Unfortunately, there is a great deal of prejudice concerning this issue, with many people believing that any such attempts cannot succeed. This is not a useful point of view to take and is, in fact, at odds with generations of successes in field theory, many-body physics, and condensed matter physics:

\section{nonperturbative does not mean intractable!}

A trivial example is provided by elementary calculus. As is well known the function $f(x)=\mathrm{e}^{-\frac{1}{x}}$ has a zero radius of convergence and hence no sensible 'perturbation theory'. However, if a sufficiently clever theorist were able to derive the 'Dyson-Schwinger' differential equation $f^{\prime}(x)=\frac{1}{x^{2}} f(x)$, almost anyone could then derive any desired property of $f$.

More substantial examples are provided by the Gell-Mann - Brueckner resolution to the infrared divergence problem in the degenerate electron gas, the Galitskii method for dealing with strong short range repulsive interactions in nuclear physics, and Laughlin's explanation of the fractional quantum Hall effect.

The point is this: QCD is described by a Lagrangian and therefore all of the properties of QCD are carried by its diagrams. To make progress nonperturbatively one must find an infinite set of diagrams to 'sum' which capture the majority of the physics one is interested in. It would be a completely new occurrence in physics if this approach should not work for confinement, requiring the introduction of a concept such as intrinsic incomputability' (with commensurate implications for attempts at deriving the 'theory of everything').

In the following a very brief summary of Dyson-Schwinger and Green's function approaches to confinement are presented. I shall also recall some salient features of the vortex picture of confinement. Space constraints forbid discussing many other popular ideas of confinement such as the dual superconductor picture, merons, and monopoles.

\section{The Dyson-Schwinger Formalism}

There has been much progress in the Dyson-Schwinger approach to confinement in recent years. The idea is to truncate the infinite series of Dyson-Schwinger equations (which are equivalent to the field theory) in such a way that the leading infrared behaviour of the ghost and gluon propagators may be reliably extracted. This endeavour is assisted by appealing to known properties of nonperturbative vertices, either through Slavnov-Taylor identities, the application of BRST symmetries, or properties of special gauges (in particular, the ghost-gluon vertex is not renormalized in Landau gauge). It has been found that including the ghost propagator in the coupled set of D-S equations is important. One may then solve for the infrared behaviour of the ghost and gluon propagators under various approximations or solve the coupled integral equations numerically[16]. 
A convincing demonstration of confinement results. Analytic work finds that the infrared propagators behave as

$$
Z(k) \rightarrow\left(\frac{k^{2}}{\sigma}\right)^{2 \kappa}, k \rightarrow 0
$$

and

$$
G(k) \rightarrow\left(\frac{\sigma}{k^{2}}\right)^{\kappa}, k \rightarrow 0 .
$$

with $\kappa \approx 0.6$. This value is very stable under modification of the truncations or Ansätze. Equations 6 and 7 demonstrate that the Kugo-Ojima confinement criteria are met. Furthermore, numerical solutions are in remarkable agreement with lattice results[5], indicating the utility of the truncations made.

\section{Green's Function Approach in Coulomb Gauge}

It has been 25 years since Gribov first noted that the imposition of Coulomb gauge in non-Abelian theories is beset with ambiguities [17]. The ambiguity arises because more than one solution to the gauge constraint, $\nabla \cdot \mathbf{A}=0$ may exist. Gribov showed that the condition for this to occur was that nontrivial solutions to the equation $\nabla \cdot \mathbf{D}=0$ had to exist. Here $\mathbf{D}$ is the covariant derivative in adjoint representation. Gribov proposed to resolve the ambiguity by restricting the gauge configurations to those with a positive definite value of $\operatorname{det}(\nabla \cdot \mathbf{D})$, called the Gribov region. He also noted that such a constraint imposes a boundary in field space, and this boundary can affect the gluon dispersion relation, causing confinement.

Much progress has occurred since Gribov framed his conjectures. It is now known that the Gribov region does not resolve the Gribov ambiguity, rather a smaller region called the fundamental modular region (FMR) is required. Furthermore, the FMR is contained in the Gribov region, the FMR incloses the origin, it is convex, and its boundary sometimes coincides with the boundary of the Gribov region[18]. Topological field configurations are realized through the imposition of nontrivial boundary conditions at the boundary of the FMR. Finally, Zwanziger has argued that physical matrix elements draw their support solely from the intersection of the Gribov and fundamental modular boundaries[19].

It is suggestive that the operator $\nabla \cdot \mathbf{D}$ appears in the instantaneous portion of the interaction in the QCD Hamiltonian in Coulomb gauge[21]:

$$
V_{\text {Coul }}=-\frac{1}{2} \int d^{3} x d^{3} y \mathscr{J}^{-1} \rho^{a}(\mathbf{x})\left\langle\mathbf{x} a\left|\frac{g}{\nabla \cdot \mathbf{D}} \nabla^{2} \frac{g}{\nabla \cdot \mathbf{D}}\right| \mathbf{y} b\right\rangle \mathscr{J} \rho^{b}(\mathbf{y})
$$

where $\mathscr{J}=\operatorname{det}(\nabla \cdot \mathbf{D})$ is the Faddeev-Popov determinant and the colour density is given by $\rho^{a}=-f^{a b c} \mathbf{A}^{b}(\mathbf{x}) \cdot \mathbf{E}^{c}(\mathbf{x})$. If vacuum field configurations are dominated by those near the boundary of the Gribov region there will be a strong infrared enhancement of the instantaneous interaction, which may cause confinement. This old observation has recently received support from several lattice computations[22, 23]. 
It is known that the non-Abelian Coulomb interaction is renormalization group invariant and that it generates the complete running coupling of QCD[24]. Furthermore, quarks decouple from transverse gluons in the static limit and therefore this interaction must generate confinement. Indeed, Zwanziger has recently shown that the non-Abelian Coulomb interaction provides an upper bound to the Wilson loop interaction[20] and has conjectured that this bound is saturated (numerical evidence in favour of this conjecture is provided in Ref. [22]).

It is clear that the instantaneous interaction is an important element of QCD. Recently an analytical attempt to understand this operator has been made in the Greens function approach [25]. The central idea was to compute the kernel appearing in Eq. 8 by summing all diagrams which contribute at leading order in the infrared[26]. The resulting Dyson equation was solved with the gluon propagator equation (which was obtained with the aid of a Gaussian Ansatz for the pure gauge vacuum). Numerical solution of the coupled nonlinear integral equations yielded a nontrivial gluonic quasiparticle dispersion relation and a linearly confining instantaneous interaction in remarkable agreement with lattice Wilson loop results[25]. These results are in agreement with the Kugo-Ojima confinement criteria, namely the inverse Faddeev-Popov operator (which is the Coulomb gauge analogue of the ghost propagator) is infrared enhanced. The infrared behaviour of the transverse gluon propagator is more problematic, but it is now understood to arise from strong modifications of the vacuum Ansatz near the Gribov boundary[27].

\section{Vortices}

Singular gauge transformations generate local objects of great interest. For example, zero-dimensional gauge dislocations are associated with instantons, one-dimensional with monopoles, and two-dimensional with vortices. A vortex is a sheet (in 4-d) of infinite field strength which is associated with a $Z_{N_{c}}$ singular gauge transformation (see Ref. [28] for more details). It has been postulated that vortices drive confinement [29] because they are localized field configurations which percolate the lattice. The argument is quite general and relies on the fact that localized field distributions contribute independently to the expectation value of the Wilson loop operator, and hence yield an area law interaction.

There are many other attractive features of the vortex confinement picture, for example, deconfinement may be viewed as a vortex de-percolation phase transition[31] and the vortex density has been shown to scale in maximal centre gauge, thereby establishing their physical nature[32]. Lastly, it has recently been shown that monopoles may be understood in terms of vortex self intersections, thereby introducing topological field configurations into the discussion [33].

This compelling picture is strongly supported by lattice computations permitted by recent advances in lattice gauge fixing algorithms. In particular figure 4 shows that the Wilson loop string tension is maintained if one projects onto vortex gauge configurations (upper green line) and that linear confinement disappears if vortices are removed (lower red line). 


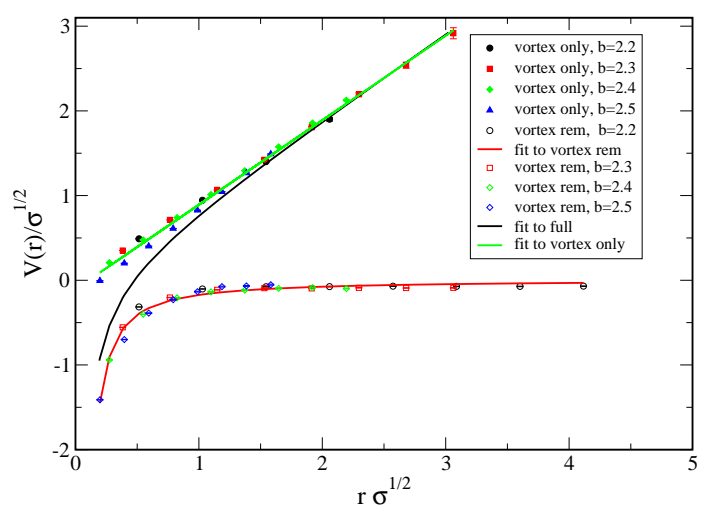

FIGURE 4. The SU(2) Wilson Loop Potential with and without Vortices[28].

\section{CONCLUSIONS}

Great strides have been made recently in the effort to understand confinement. New lattice algorithms allow a detailed study of the importance of different model gauge configurations and of the onset of string-like behaviour in flux tubes. Multiquark states are also beginning to be probed. At the same time, analytical methods are rapidly approaching the time when quantitatively accurate computations of nonperturbative phenomena are possible. And we are already seeing convincing demonstrations of the general features of confinement.

Of course much remains to be done. The analytical formalisms need to examine the robustness of the assumed truncations and the effects of topology and boundary conditions. At the same time, these approaches can check and compare with a new breed of fixed-gauge lattice results. There are many open issues in the lattice approach a well. For example, too many models of the QCD vacuum seem to be supported by the lattice and one needs to find some commonality among the different mechanisms and gauges. It is hoped and likely that issues such as the nature of baryonic flux tubes, the infrared behaviour of the Landau gauge gluon propagator, the onset of stringiness in flux tubes (is it at $1 / 2$ or 4 fermi?), and the nature of the non-Abelian Coulomb interaction will all be resolved in the short term.

\section{ACKNOWLEDGMENTS}

I am grateful to the organizers of Hadron03 for the invitation to speak on such a fascinating topic and for providing a wonderful venue for discussing hadronic physics. I wish to thank Reinhard Alkofer, Pierre van Baal, Jeff Greensite, Hugo Reinhardt, Adam Szczepaniak, and Daniel Zwanziger for many illuminating discussions on this topic. This work was supported by the DOE under contracts DE-FG02-00ER41135 and DEAC05-84ER40150. 


\section{REFERENCES}

1. J. Greensite, "The confinement problem in lattice gauge theory", Prog. Part. Nucl. Phys. 51, 1 (2003).

2. K.J. Juge, J. Kuti, and C.J. Morningstar, Nucl. Phys. Proc. Suppl. 63, 326 (1998).

3. T. Kugo and I. Ojima, Prog. Theor. Phys. Suppl. 66, 1 (1979).

4. T. Kugo, arXiv:hep-th/9511033

5. R. Alkofer and C. S. Fischer, "The Kugo-Ojima confinement criterion and the infrared behavior of Landau gauge QCD", arXiv:hep-ph/0309089

6. D. Zwanziger, Nucl. Phys. B 485, 185 (1997).

7. G. S. Bali, K. Schilling and C. Schlichter, Phys. Rev. D 51, 5165 (1995).

8. G. S. Bali, K. Schilling and A. Wachter, Phys. Rev. D 56, 2566 (1997).

9. G. S. Bali, Phys. Rev. D 62, 114503 (2000).

10. C. J. Morningstar, K. J. Juge and J. Kuti, Nucl. Phys. Proc. Suppl. 73, 590 (1999); K. J. Juge, J. Kuti and C. Morningstar, Phys. Rev. Lett. 90, 161601 (2003).

11. M. Luscher and P. Weisz, JHEP 0207, 049 (2002).

12. H. Ichie, V. Bornyakov, T. Streuer and G. Schierholz, "The flux distribution of the three quark system in SU(3)", arXiv:hep-lat/0212024

13. C. Alexandrou, P. De Forcrand and A. Tsapalis, Phys. Rev. D 65, 054503 (2002).

14. T. T. Takahashi, H. Suganuma, Y. Nemoto and H. Matsufuru, Phys. Rev. D 65, 114509 (2002).

15. F. Okiharu and R. M. Woloshyn, "A study of colour field distributions in the baryon", arXiv:hep-lat/0310007

16. P. Watson and R. Alkofer, Phys. Rev. Lett. 86, 5239 (2001); C.S. Fischer, R. Alkofer, and H. Reinhardt, arXiv:hep-ph/0202195. C. Lerche and L. von Smekal, arXiv:hep-ph/0202194 D. Atkinson and J.C. Bloch, Phys. Rev. D58, 094036 (1998); D. Zwanziger, arXiv:hep-th/9410019.

17. V.N. Gribov, Nucl. Phys. B139, 1 (1978).

18. P. Van Baal, "The QCD Vacuum", arXiv:hep-lat/9709066 "Nonperturbative Analysis, Gribov Horizons, and the Boundary of the Fundamental Domain", arXiv:hep-lat/9208027

19. D. Zwanziger, "Non-perturbative Faddeev-Popov formula and infrared limit of QCD", arXiv:hep-ph/0303028

20. D. Zwanziger, Phys. Rev. Lett. 90, 102001 (2003).

21. N. H. Christ and T. D. Lee, Phys. Rev. D 22, 939 (1980).

22. A. Cucchieri and D. Zwanziger, Phys. Rev. D 65, 014001 (2002).

23. J. Greensite and S. Olejnik, Phys. Rev. D 67, 094503 (2003).

24. D. Zwanziger, Nucl. Phys. B 518, 237 (1998).

25. A. P. Szczepaniak and E. S. Swanson, Phys. Rev. D 65, 025012 (2002).

26. E. S. Swanson and A. P. Szczepaniak, "Constructing confinement", arXiv:hep-ph/0205079

27. A. P. Szczepaniak, "Confinement and gluon propagator in Coulomb gauge QCD", arXiv:hep-ph/0306030

28. K. Langfeld, "Vortex Matter in SU(3) Lattice Gauge Theory", arXiv:hep-lat/0307030

29. G. 't Hooft, Nucl. Phys. B 153, 141 (1979); J.M. Cornwall, Nucl. Phys. B 157, 392 (1979).

30. K. Langfeld, "Vortex induced confinement and the Kugo-Ojima confinement criterion", arXiv:hep-lat/0204025

31. K. Langfeld, Phys. Rev. D 67, 111501 (2003).

32. See K. Langfeld, H. Reinhardt, and O. Tennert, Phys. Lett. B419, 317 (1999). We note that the story is not simple since density scaling is not seen in laplacian gauge. Furthermore, only $62 \%$ of the SU(3) string tension (as opposed to all of the $\mathrm{SU}(2)$ string tension) is recovered upon vortex projection in the maximal centre gauge [28] .

33. H. Reinhardt, Nucl. Phys. B 628, 133 (2002). 